\title{
Rapid demand curves for behavioral economics
}

\author{
THOMAS G. RASLEAR, RICHARD A. BAUMAN, STEVEN R. HURSH, \\ DAVID SHURTLEFF, and LAURENCE SIMMONS \\ Walter Reed Army Institute of Research, Washington, D.C.
}

\begin{abstract}
A method for determining the relationship between food consumption and the price of food (demand function) in behavioral economic experiments is described. Although previous methods have generally required as long as $\mathbf{4 0}$ days, the present method can generate a complete demand function within 7 days, and therefore may be more suitable for use in the evaluation of drugs, toxins, and physiological/anatomical interventions. Moreover, measures of circadian rhythmicity, postreinforcement pause durations, and interresponse times can also be generated. Three experiments tested the stability of the method in a variety of procedural manipulations: repeated exposure to the procedure, increasing versus random daily food price, and size of daily changes in food price. The procedure generated demand functions that were similar to those that require more extended testing, and the demand functions were not generally affected by procedural manipulations. Body weight, which can also affect consumption, generally decreases with increases in the price of food; so this variable should be recorded and used as a covariate in analyzing demand functions. With the exception of circadian rhythmicity, the other measures were stable across procedural variations and showed expected changes as a function of food price: postreinforcement pause durations increased as price increased, but interresponse times did not.
\end{abstract}

Recently a great deal of interest has focused on the application of economic principles to the analysis of behavioral data generated by animal subjects (Allison, 1983; Hursh, 1980, 1984). A primary task for such an analysis is the generation of a function that relates the price of a commodity (e.g., the number of responses required to obtain a 45-mg food pellet) to the quantity of the commodity that is consumed. Functions that relate quantity consumed to price are known in economics as demand curves, and Figure 1 illustrates the features of demand curves that are commonly seen in the behavioral literature. Assume that the subjects earn all of their food within the experimental apparatus by performing a specified response, and that the delivery of food pellets is contingent upon production of a fixed number of responses (i.e., a fixed ratio, or FR, schedule of reinforcement). Price $(P)$, then, is measured in responses per pellet, as indicated along the abscissa of Figure 1 . The quantity consumed $(Q)$, measured in pellets of food, is plotted on the ordinate. Note that the ordinate and abscissa are both logarithmic. A demand curve, such as $A$ in Figure 1, is generated by changing the price of the commodity and measuring the quantity of the commodity that is then consumed. Demand elasticity is defined as the percentage change in consump-

The research described in this report was conducted in compliance with the Animal Welfare Act and other Federal statutes and regulations relating to animals and experiments involving animals, and adheres to the principles stated in the Guide for the Care and Use of Laboratory Aniamls, NIH publication 85-23. The views of the author(s) do not purport to reflect the position of the Department of the Army or the Department of Defense, (para 4-3, AR 360-5). The authors' mailing address is Department of Neurosciences, Walter Reed Army Institute of Research, Washington, DC 20307-5100. tion per percentage change in price $(\% \Delta Q / \% \Delta P)$. In double logarithmic plots, the slope of the demand curve can be directly read as elasticity. Unit elasticity is depicted in curve $B$, since a $1 \%$ change in price produces a $1 \%$ change in consumption (i.e., slope $=-1$ ). Inelastic demand is illustrated in curve $A$, since the slope of the curve is $>-1$, and elastic demand (slope $<-1$ ) is illustrated in curve C (consult Hursh, 1980, for details).

The analysis of demand curves can reveal fundamental information concerning the economic conditions of an experiment (see Hursh, 1984). For instance, the relative elevation in quantity consumed at the origin (intensity of demand) appears to vary with changes in such "motivational" variables as level of deprivation and magnitude of reinforcement, whereas changes in elasticity of demand are associated with the nature of the commodity being worked for, the species of the consumer, the availability of substitutes, and the economic context of the experiment (i.e., open vs. closed economy; see Bauman, Raslear, Hursh, Shurtleff, \& Simmons, 1986; Elsmore, Fletcher, Conrad, \& Sodetz, 1980; Hursh, 1980, 1984; Hursh \& Natelson, 1981).

Given the wide variety of variables that appear to affect demand curves, our lab has begun to explore the suggestion raised by Hursh (1984) that behavioral economics might provide a useful tool for the evaluation of drugs, toxicants, and physiological/anatomical interventions. A major obstacle, however, is the fact that the generation of a demand curve typically requires a relatively long period of time. For instance, the original method described by Hursh required 5 to 6 months to generate a function with 5 points, and a faster method reduced this to only 40 days. However, if several levels of a treatment are to 
DEMAND CURVES

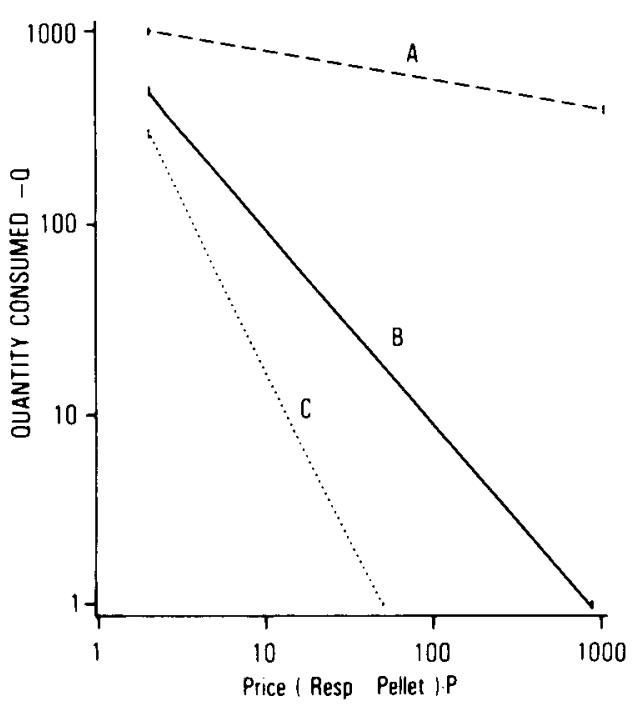

Figure 1. Three plots of quantity consumed $(Q)$ as a function of price $(P)$ in $\log -\log$ coordinates. The slopes of the functions show the percentage change in consumption per percentage change in price, which is defined as demand elasticity. Unit elasticity (slope $=-1$ ) is depicted in curve B since a 1\% change in price produces a 1\% change in consumption. Inelastic demand is illustrated in curve $A$ (slope $>-1$ ), and elastic demand (slope $<-1$ ) is illustrated in curve $\mathbf{C}$.

be given (e.g., to evaluate dose-response relationships), or if replications are needed, even a 40-day period is too time-consuming to be practical.

This paper describes a method for generating demand curves within 7 days. In addition to providing the necessary data for an economic analysis, the method is capable of providing information about circadian rhythmicity, postreinforcement pause (PRP) durations, and interresponse times (IRTs). Three experiments are reported that demonstrate the stability of the procedure over time, and the invariance of the demand curve under noneconomic procedural manipulations. In Experiment 1, stability over time of the various measures of performance was examined by treating the animals as if they were in an ABBA experimental design. Since the treatment conditions, $A$ and $B$, are identical, differences in performance that are due either to "treatment" effects or to the sequence of exposure should reflect upon the procedure's stability over time. In Experiment 2, the effect on performance measures of progressive daily increases in price versus random daily changes in price was examined. Finally, in Experiment 3, the effect of the size of daily changes in price was examined.

\section{GENERAL METHOD}

\section{Subjects}

All subjects were male albino Sprague-Dawley derived rats, 90-120 days old, from the Walter Reed colony. All animals were experimentally naive. Prior to the experiment, the rats were individually housed, for 7 to 14 days, in rack-mounted hanging wire cages in the same room in which the experiment was conducted.

\section{Apparatus}

Behavioral measurements were conducted in six modified hanging wire cages (Wahmann Co., Baltimore, MD). Each cage was $24.1 \mathrm{~cm}$ long, $20.3 \mathrm{~cm}$ wide, and $18.4 \mathrm{~cm}$ deep. A single response lever, water bottle, and food magazine were mounted on the fron of each cage. The response lever was $5.1 \mathrm{~cm}$ long, $1.3 \mathrm{~cm}$ wide, and extended $1.3 \mathrm{~cm}$ into the cage. The lever and food magazine were mounted $3 \mathrm{~cm}$ apart on the center of the cage front, $5.1 \mathrm{~cm}$ above the floor. The water bottle spout extended into the cage at the level of the magazine and lever on the same side of the cage as the magazine, $4 \mathrm{~cm}$ from it. Food pellet dispensers (Gerbrands, Model G5100) were mounted above each cage. These delivered 45$\mathrm{mg}$ food pellets (Bio-Serv, Frenchtown, NJ) whenever a specified number of responses was made on the associated lever. The food pellets were the only source of food available to the rats during the experiment.

When applied to the lever, a force equivalent to $27 \mathrm{~g}$ caused a microswitch mounted on the lever to close, and this defined a response. Responses were sensed by a PDP-8/e computer (Digital Equipment Co.) that was located outside the experimental chamber. The computer operated the pellet dispensers and recorded responses, the time of day at which responses occurred, and the time between successive responses.

The experimental chamber was a shielded room (Shieldtron Inc., Moorestown, $\mathrm{NJ}$ ), $2.3 \mathrm{~m}$ high, $2.4 \mathrm{~m}$ wide, and $5.4 \mathrm{~m}$ long, that contained the cage rack on which the experimental and other cages were hung, as well as several other pieces of equipment. Temperature in the room was maintained at approximately $21^{\circ} \mathrm{C}$, and the relative humidity was $50 \%$. A 12:12-h light-dark cycle was maintained in the room (lights on at $0600 \mathrm{~h}$, off at $1800 \mathrm{~h}$ ).

\section{Procedure}

The rats were acclimated to the experimental chamber and the light-dark cycle for a minimum of 1 week prior to use. At the beginning of the experiment, the rats were placed in the experimental cages where they remained until the end of the experiment. During that period of time, all food was obtained by leverpresses, which allowed for computer monitoring of (1) daily activity patterns (responses and food pellets delivered during the light and dark phases of the light-dark cycle), (2) the latency of the first response following a food pellet delivery (that is, the PRP), and (3) the time between successive leverpresses (that is, the IRT), exclusive of the PRP. Each rat was weighed daily between 1000 and $1200 \mathrm{~h}$.

The number of responses required to obtain a single food pellet (FR) was changed at $0600 \mathrm{~h}$ each day for the duration of the experiment (generally 7 days). The first FR in each experiment was always 1.0 . This was done to give the rats the opportunity to acquire the lever response. Data generated from this first FR was not used, in order to allow for individual differences in learning to barpress. Each FR schedule was continuously available throughout a 24-h period.

\section{Data Analysis}

Demand curves, plots of food consumed (pellets) as a function of the price of food (FR), were constructed from 24-h pellet counts at each $F R$ value.

For subsequent computer analysis, PRPs and IRTs were accumulated for each 24-h period in 3.0- and 0.1-sec bins, respectively. PRPs were not included in IRT distributions. For use in generating plots and statistical analyses, medians and interquartile ranges of each measure were then determined for each rat. These measures are intended to provide an index of the integrity of motor response function (see General Discussion).

The number of food pellets obtained in the light and the dark was also monitored for each rat in order to provide an index of circadian rhythmicity. The measure used was the proportion of pellets obtained in the dark (pellets obtained in dark/total pellets). 
Statistical analyses were conducted using the SAS (SAS Institute Inc., 1985) and BMDP statistical packages (Dixon, 1981). Unless otherwise specified, all analyses were repeated measures analyses of variance (ANOVAs). Sphericity tests were routinely performed on the data by the BMDP program ( $\mathrm{P} 2 \mathrm{~V})$, and, in those cases where sphericity was violated, we report the tail probabilities calculated from the Greenhouse-Geisser adjustment to degrees of freedom. For reliable effects $(p<.05$ ), a measure of effect strength (proportion of variance accounted for, $\omega^{2}$, see Hays, 1963) was also calculated.

\section{EXPERIMENT 1}

A major procedural concern for any rapid behavioral methodology is whether the data is representative of performance in a similar task when longer periods of time are allowed for stabilization of the behavior. The first experiment addresses this issue by examining performance in the procedure over a 28-day period. During that period, the basic procedure was repeated four times so that changes due to experience with the procedure (e.g., sequential effects, practice effects, etc.) could be detected. Four replications were performed so that the data could be examined as if an ABBA ( $=$ baseline, $B=$ treatment) experimental design were in use.

\section{Method}

The subjects (6 rats) and the apparatus were as described in General Methods.

At the beginning of the experiment, the rats were placed in the experimental cages, where they remained for the next 28 days. Over the 28 days of the experiment, the number of responses required to obtain a single food pellet (i.e., the FR) was changed each day by repeating the following sequence of FRs four times: $1,1,15$, $45,90,180,360$. Data from the first FR $I$ in each replication was not used.

\section{Results}

Figure 2A presents the mean total pellets consumed as a function of price (FR) for the four replications of the FR series. Unlike the curves in Figure 1, the functions depicted in Figure $2 A$ are demand curves of a "mixed" type (i.e., elasticity or slope varies as a function of price). Although the functions for each replication overlap considerably, there is some indication that consumption increased as the animals were repeatedly exposed to the FR series. Analysis of the demand functions indicated that there were significant effects of $\operatorname{FR}[F(5,25)=71.5, p=$ $\left..0000, \omega^{2}=.25\right]$, and of the interaction of treatments and replications $\left[F(1,5)=12.78, p=.0159, \omega^{2}=.012\right]$. The strong effect of variations in price on consumption are, of course, expected. The reliable treatment $\times$ replications effect indicates that some systematic change has occurred across the four replications of the FR series, but it should be noted that this effect, as indicated by $\omega^{2}$, is relatively weak. Since young animals were used, it is possible that systematic weight gains over a 28-day period could have occurred, and such weight gains would correlate with increases in consumption.

Figure 2B presents the mean body weights of the rats for the 28 days of the experiment, and here it is obvious that body weight changed as a function of time in the experiment. Analysis indicated that there were significant effects of the treatments [Weeks 1 and 4 vs. Weeks 2 and $\left.3, F(1,5)=23.64, p=.0046, \omega^{2}=.00004\right]$, of the replications [Weeks 1 and 2 vs. Weeks 3 and $4, F(1,5)=$ $\left.39.95, p=.0015, \omega^{2}=.0028\right]$, of the treatments $\times$ replications interaction $\left[F(1,5)=15.5, p=.011, \omega^{2}=\right.$ $.00026]$, and of the treatments $\times$ replications $\times$ FR in teraction $\left[F(5,25)=5.23, p=.037, \omega^{2}=.00012\right]$. These factors, although reliable, are not strongly predictive of changes in body weight.

As noted above, since increases in body weight correlate strongly with increases in consumption, it is possible that the changes in consumption seen in Figure $2 \mathrm{~A}$ can be attributed to changes in body weight. This possibility was statistically assessed by correlating food consumption and body weight across experimental conditions. Although the correlation was statistically reliable $[r=$ $0.2423 ; F(1,142)=8.86, p=.0034]$, the coefficient of determination $\left(r^{2}\right)$ indicated that only $5.87 \%$ of the variance in consumption could be accounted for by body
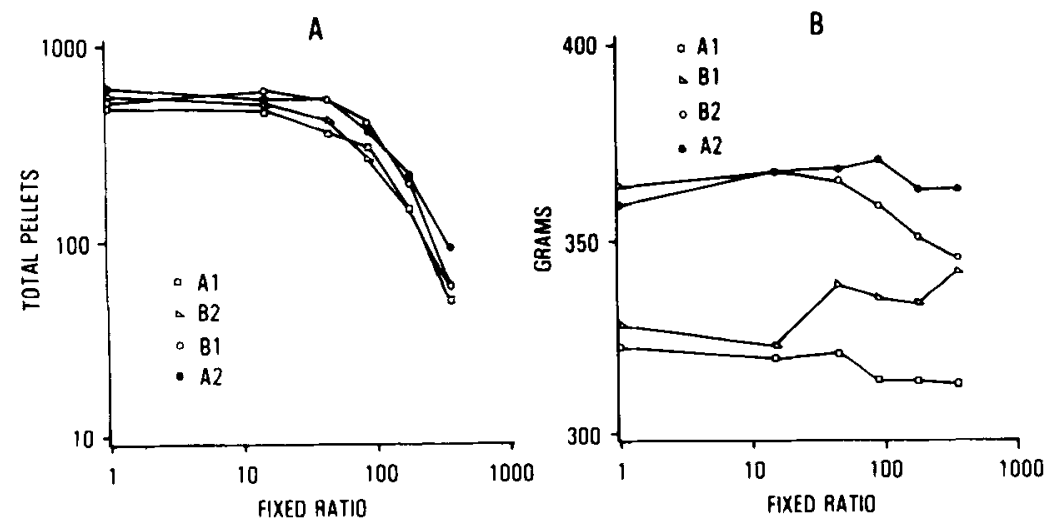

Figure 2. Panel A: Mean total pellets consumed as a function of the fixed ratio (price) over four successive repetitions of the procedure in a simulated ABBA design. Panel B: Mean body weight in grams as a function of the fixed ratio in the simulated ABBA design. 
A

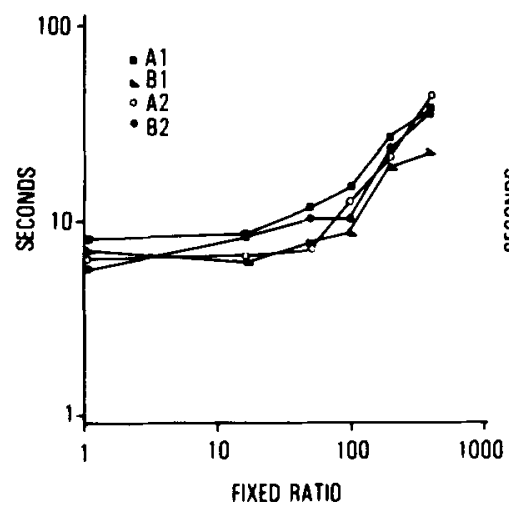

B

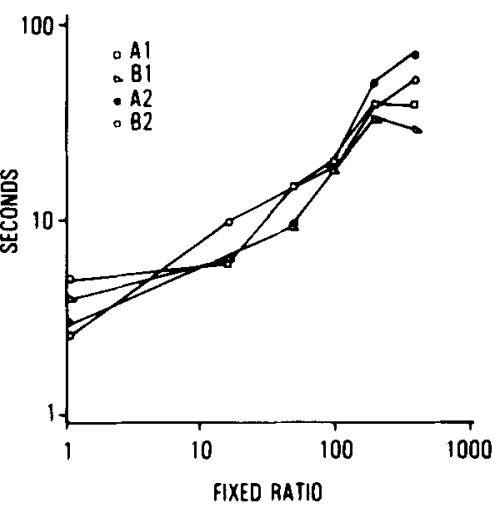

Figure 3. Panel A: Mean median postreinforcement pause as a function of the fixed ratio in the simulated ABBA design. Panel B: Mean interquartile range of the postreinforcement pause as a function of the fixed ratio in the simulated ABBA design.

weight. Clearly, then, of the factors that potentially control changes in food consumption, the FR is the strongest, accounting for $25 \%$ of the variance in pellet consumption.

Figure 3 presents the mean median PRP and mean interquartile range of the postreinforcement pause (IQPRP) as a function of the price of food. As expected from studies of FR pausing in traditional "open economy" operant conditioning experiments (e.g., Felton \& Lyon, 1966; Mazur, 1983), both the PRP and the IQPRP increase as a function of the FR [respectively, $F(5,25)=5.15, p=$ $.0491, \omega^{2}=.3416$, and $F(5,25)=16.70, p=.0000$, $\left.\omega^{2}=.552\right]$. There were no other reliable effects.

Figure 4 presents the mean median IRT and mean interquartile range of the interresponse time (IQIRT) as a function of the price of food. Although the third replication of the FR series shows some tendency for longer IRTs as the FR increases, there were no reliable effects of the FR, or of any other variable for IRTs or IQIRTs.

Figure 5 presents the mean proportion of pellets obtained during the dark as a function of the price of food, which is intended as a measure of circadian rhythmicity. In each replication of the FR series, there is a clear tendency for the animals to obtain more of their food during the dark. An ANOVA indicated a reliable FR effect $\left[F(5,25)=16.71, p=.0000, \omega^{2}=.009\right]$, as well as a reliable replication $\times$ treatment effect $[F(1,5)=6.61$, $p=.05, \omega^{2}=.0012$ ]. Clearly, experience with the FR series and the abrupt transition from very high FR values to low FRs affects the feeding patterns of the animals in a complex manner.

\section{Discussion}

The four demand curves in Figure $2 \mathrm{~A}$ are each qualitatively of the same form as those produced by Hursh (1980) over a longer period of time. Changes in body weight may be a factor in producing changes in the demand for food (or vice versa), but, at most, body weight can account for less than $6 \%$ of the variance in demand, and sequential and/or experiential factors (presumably operating through the variable of treatments $x$ replications) can account for approximately $1 \%$ of the variance in demand
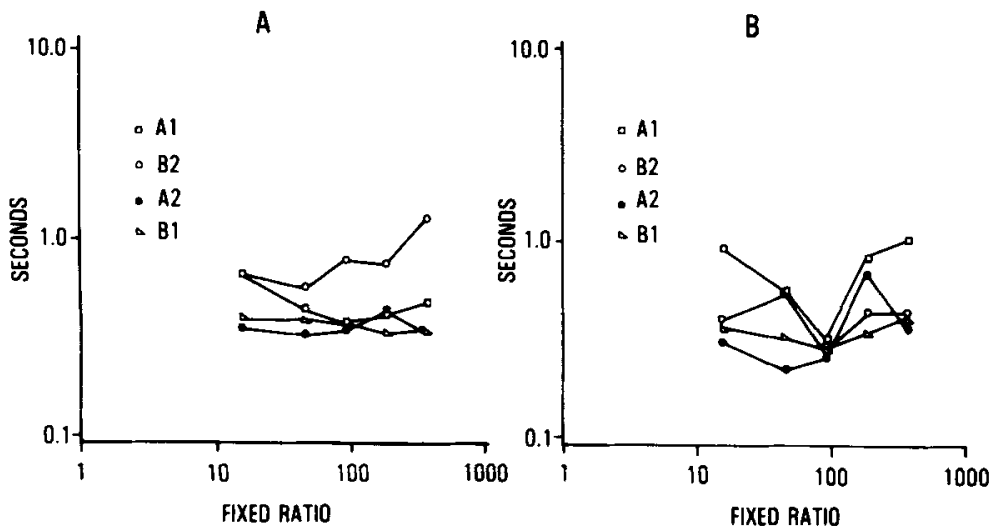

Figure 4. Panel A: Mean median interresponse time as a function of the fred ratio in the simulated ABBA design. Panel B: Mean interquirtile range of the interresponse time as a function of the fixed ratio in the simulated ABBA design. 


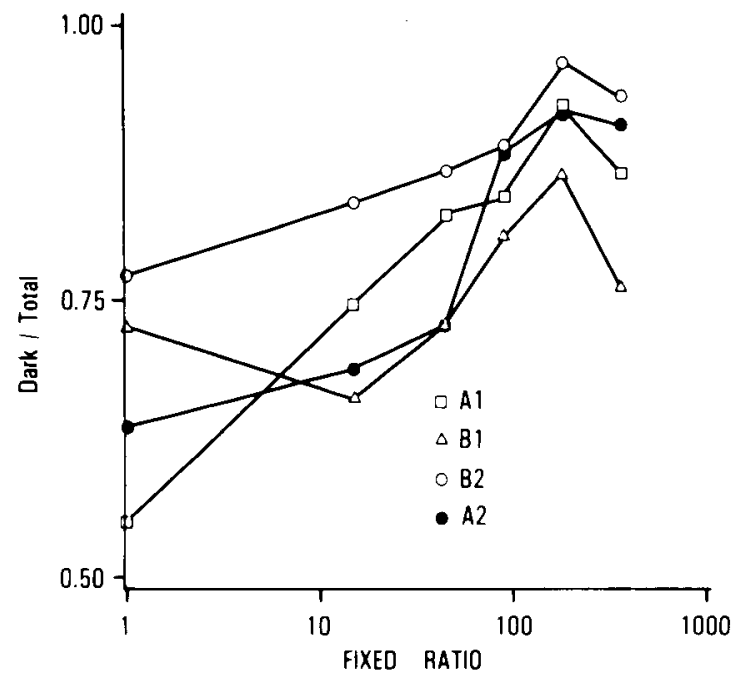

Figure 5. Mean proportion of pellets earned in the dark as a function of the fixed ratio in the simulated ABBA design.

for food. Variations in the FR value, on the other hand, account for $25 \%$ of the variance in demand for food. There is, therefore, no doubt that the present method can reliably produce demand curves that are similar in form to those generated over a longer time frame and that represent a direct effect of manipulations in price (FR). Moreover, the changes in body weight and in consumption that occurred over the 28 days of the experiment suggest that a more rapid procedure may possess an advantage over longer procedures, namely, the ability to measure consumption as a function of price in the absence of significant weight changes.

Previous studies of IRTs and PRPs in FR schedules have reported that PRPs increase as a function of the FR, but that IRTs of less than $1 \mathrm{sec}$ remain relatively constant (Felton \& Lyon, 1966; Mazur, 1983). Those studies, however, were all performed in an open economy: the animals were food deprived, earned some portion of their food within a short experimental session, and were consequently returned to their home cages where supplemental food was provided. The present experiment demonstrates that the functional relationships between FR value, PRP, and IRT also hold for the "closed economy" experiment and for much larger FR values than have been previously reported. Moreover, the lack of reliable differences as a function of repeated exposures indicates that a valid measurement can be obtained within a single day's exposure to each FR.

The measure of circadian rhythmicity, on the other hand, is clearly affected by repeated exposures to the FR series. Repetitions of the FR series itself or changes in body weight may have been causing a change in eating patterns. However, none of these factors was strongly predictive of rhythmicity. This indicates that the current ABBA design may be suitable for experiments in which a stable measure of circadian function is desired, but that it should be used cautiously.

\section{EXPERIMENT 2}

Experiment 1 demonstrated that the most important factor controlling variations in the demand curves with repeated exposures to the FR series was the FR series. However, it is possible that a different result would be obtained if the FRs were not presented in an ascending series. If there were sequential effects within an FR series, then the demand function would have to be derived from a method that eliminated or minimized such effects. Experiment 2 examined this issue by comparing an FR series presented in an ascending order to one group of rats with the same FR series presented in a random order to a different group of rats.

\section{Method \\ The subjects (12 rats) and the apparatus were as described in General Methods. \\ Two groups of 6 rats each were successively studied over a 14- day period. Each group of rats contained 3 rats that were assigned to the ascending FR condition and 3 rats that were assigned to the random FR condition. Rats in the two conditions were assigned to alternate cages. Each rat in the random FR condition was exposed to a different random order of FRs. As previously, on the first day in the experimental cages all the rats were given an FR 1 require- ment to allow for acquisition of barpressing for food, and data from that FR 1 was discarded. The ascending FR series was: 1, 15, 45, $90,180,360$. The sequences used for each rat in the random con- dition are presented in Table 1.}

\section{Results and Discussion}

Figure $6 \mathrm{~A}$ presents the mean body weights of the two groups as a function of the FR value. Unlike in Experiment 1 (see Figure 2B), where there appeared to be no systematic change in body weight as a function of FR value, in Experiment 2, there was a clear trend for body weight to decrease as the FR was increased in both groups. An ANOVA indicated that there was no significant group effect or group $\times$ FR interaction, but that there was a reliable FR effect $\left[F(5,45)=13.25, p=.0001, \omega^{2}=\right.$ $.0005]$.

Figure $6 \mathrm{~B}$ presents the mean demand curves for the two conditions as a function of the FR value. The demand curves for the two groups in Figure 6B do not appear to be dramatically different, although there is some tendency for the random group to more rapidly decrease consumption. An ANOVA indicated that there was no group or group by FR interaction, but there was a reliable FR effect $\left[F(5,45)=49.85, p=.0000, \omega^{2}=.46\right]$.

Table 1

Sequence of Fixed Ratios for Rats in the Random Condition

\begin{tabular}{crrrrrr}
\hline & \multicolumn{7}{c}{ Rats } \\
\cline { 2 - 7 } Order & \multicolumn{1}{c}{1} & \multicolumn{1}{c}{2} & \multicolumn{1}{c}{3} & \multicolumn{1}{c}{4} & \multicolumn{1}{c}{5} & \multicolumn{1}{c}{6} \\
\hline 1 & 360 & 1 & 1 & 15 & 1 & 360 \\
2 & 1 & 360 & 15 & 45 & 45 & 90 \\
3 & 45 & 180 & 90 & 1 & 90 & 45 \\
4 & 15 & 15 & 180 & 360 & 360 & 180 \\
5 & 180 & 90 & 45 & 90 & 15 & 1 \\
6 & 90 & 45 & 360 & 180 & 180 & 15 \\
\hline
\end{tabular}



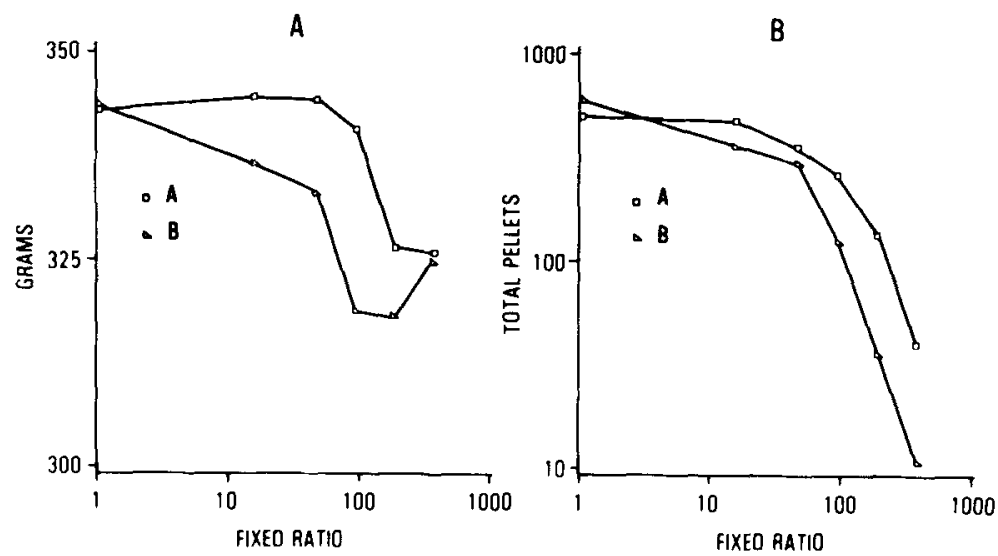

Figure 6. Panel A: Mean body weight as a function of the fixed ratio for the group given an ascending series of fixed rations (A) and for the group given a random series of fixed ratios (B). Panel B: Mean total pellet consumption as a function of the fixed ratio for the control and random groups.

Since the decrease in consumption corresponds to the decrease in body weight at the higher FRs, it is possible that changes in body weight or motivation, rather than price, could account for the decline in consumption. Unlike in Experiment 1, where there were reliable interaction effects that precluded an analysis of covariance (ANCOVA) to determine the effect of FR variations on pellet consumption that was adjusted for changes in body weight, in Experiment 2, an ANCOVA could be properly performed on the pellet consumption data, with body weight as the covariate. That ANCOVA indicated that the expected effect of price (FR) on consumption was still reliable $\left[F(5,44)=20.2, p=.0001, \omega^{2}=.47\right]$, but that there were no reliable group or group $\times$ FR effects.

As was seen in Experiment 1, there was a significant FR effect on the PRP $[F(5,45)=8.66, p=.0011$, $\omega^{2}=.39$ ], but no effect of the order of the FR series. There was a reliable group $\times$ FR interaction $[F(5,45)=$ $3.31, p=.0481, \omega^{2}=.12$, which was probably due to a lower median PRP for the random group at FR 360. The analysis of the IQPRP did not show a reliable group or group $\times$ FR effect, but did indicate that price, again, had a reliable effect on the interquartile range of the PRP $\left[F(5,45)=13.25, p=.0004, \omega^{2}=.42\right]$. This suggests that the group $\times$ FR effect seen in the median PRP might not prove to be consistent. The median IRT is, again, invariant across price. There were no reliable effects of group, FR, or group $\times$ FR for either the IRT or IQIRT.

Figure 7 presents the mean proportion of pellets obtained in the dark for the two groups as a function of the price of food. Unlike in Experiment 1, there were, in Experiment 2, no consistent change in circadian rhythmicity as a function of FR and no apparent group $\times$ FR effects. However, it would appear that the random series group was consistently less nocturnal than the ascending series group. The ANOVA, however, failed to find that any of the effects were reliable. The failure to find a reliable group difference was probably due to more variability in the random group (ascending series, $S D=0.08$; random series, $S D=0.29$ ).

The comparison of the ascending and random FR series indicates that, with the possible exception of the PRP, there are no important sequential effects associated with the ascending FR series. Once again, the importance of controlling or measuring body weight on a daily basis has been manifested, but, even when variations in pellet consumption are adjusted for body weight, the demand func-

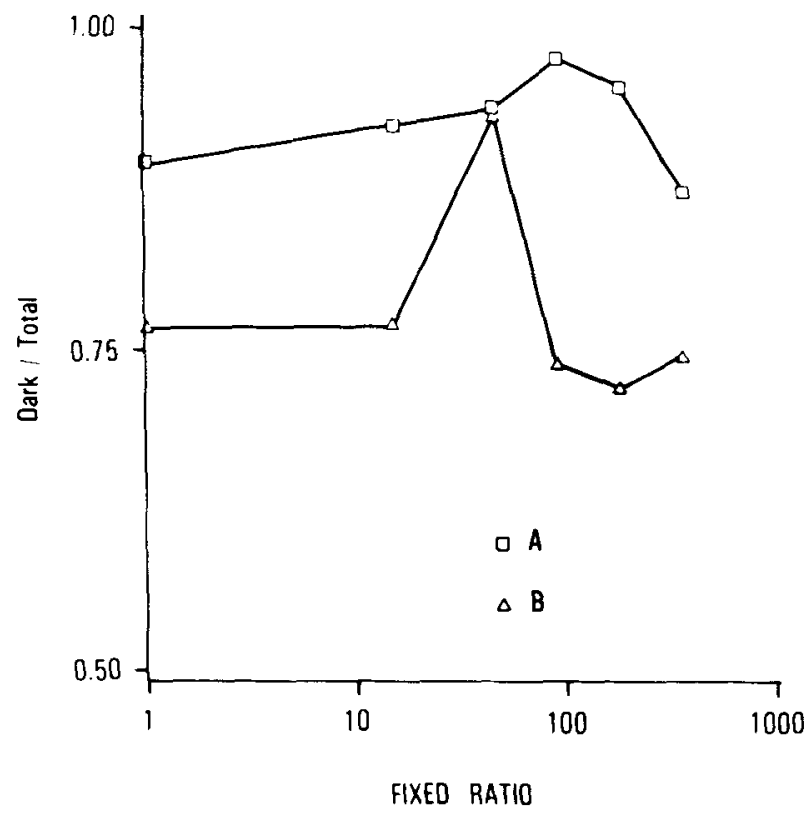

Figure 7. Mean proportion of pellets earned in the dark as a function of the fixed ratio for the control (A) and random (B) groups. 
tions for the ascending and random presentations of the FRs are similar.

\section{EXPERIMENT 3}

Spacing between stimuli has an acknowledged position within psychology as an important methodological variable (e.g., see Guilford, 1954). However, on purely economic grounds, it would be expected that such a manipulation would not change the relationship between consumption and price. Experiment 3 assessed the invariance of demand functions using two FR series that differed with respect to the step size between successive FRs.

\section{Method}

The subjects (12 rats) and the apparatus were as described in General Methods.

Each group of 6 rats was tested separately with the appropriate FR series. For Group A, the series of FR values was 1, 1, 3, 9, $27,81,243$; for Group B, the series of values was 1, 1, 15, 45, $90,180,360$. All other procedural details were as previously described.

\section{Results and Discussion}

Figure 8 presents the mean body weights and mean total pellets consumed as a function of price for Groups A and $B$. It is obvious that differences between groups exist that might be the result of the different FR series. For instance, in Group A there is a tendency for weight to increase before decreasing, whereas in Group B a steady decline is apparent. With the exception of the FR 1 points, the two groups do not have any values of the independent variable in common. Thus, the previously used methods of analysis cannot be applied. Instead, a multiple regression/correlation analysis (Cohen, 1982) was performed using the SAS GLM procedure. That analysis indicated that there was no reliable main effect of the different series or interaction with FR of the different series on pellet consumption. There was a reliable effect, however, of the FR $[F(1,68)=150.63, p=.0001$, $\left.\omega^{2}=.67\right]$.

A similar analysis of the changes in body weight indicated that only FR had a reliable effect $[F(1,68)=27.48$, $\left.p=.0001, \omega^{2}=.28\right]$. Since there were no reliable interaction effects, pellet consumption as a function of FR was reanalyzed with body weight as a covariate. That analysis indicated that the effect of $\mathrm{FR}[F(1,69)=89.01$, $p=.0001, \omega^{2}=.38$ ] on pellet consumption adjusted for body weight was still reliable.

The mean median PRP and mean median IRT functions were similar for each group and resemble PRP and IRT functions obtained in the other experiments. A multiple regression analysis indicated that only FR had a reliable effect on PRP $\left[F(1,68)=41.21, p=.0001, \omega^{2}=.36\right]$. However, the different series did have a small, but reliable, effect on IRT $\left[F(1,68)=4.18, p=.0448, \omega^{2}=\right.$ .03].

Figure 9 presents the mean proportion of pellets obtained in the dark for the two groups. Again, there is a clear tendency for the function to increase as the price of food increases, and this trend is present in both groups. A multiple regression analysis indicated that only FR had a reliable main effect $\left[F(1,68)=5.96, p=.0173, \omega^{2}\right.$ $=.08]$. This effect was present in Experiment 1 but not in Experiment 2.

\section{GENERAL DISCUSSION}

\section{Demand Curves}

The progressive decline in consumption with increasing FR values is called a demand curve because of its similarity to demand curves described in microeconomics (Samuelson, 1976; Watson \& Holman, 1977). The demand law states that, in nearly all cases, consumption of a commodity declines with increases in price. For economists, price is defined as unit cost in monetary terms. In animal experiments that do not involve money, an analogy has been drawn between monetary cost and
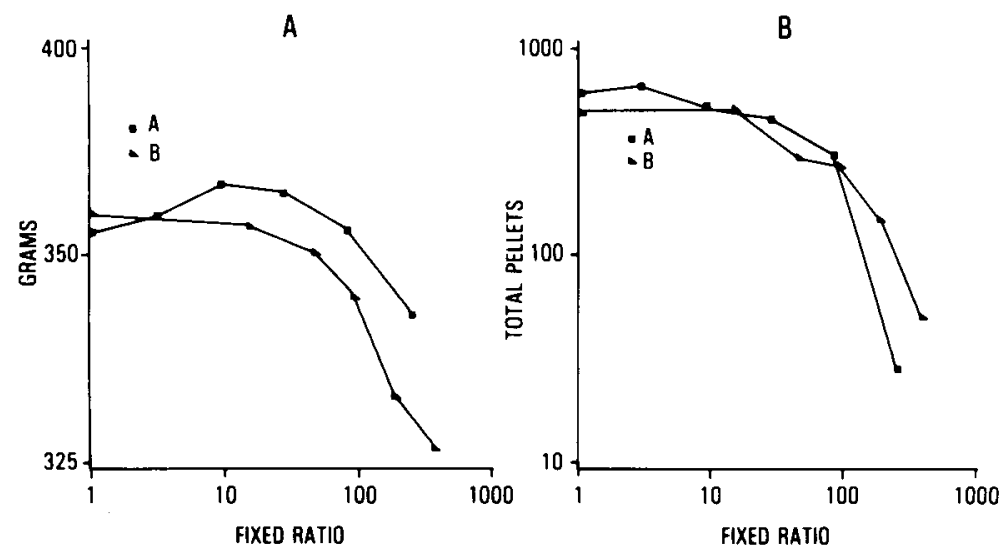

Figure 8. Panel A: Mean body weight as a function of the fixed ratio for groups of rats given different daily increments in fixed ratio. Group $A$ received the FR series 1 , $1,3,9,27,81,243$; Group B received the FR series 1, 1, 15, 45, 90, 180, 360. Panel B: Mean total pellet consumption as a function of the fixed ratio for Groups $A$ and $B$. 


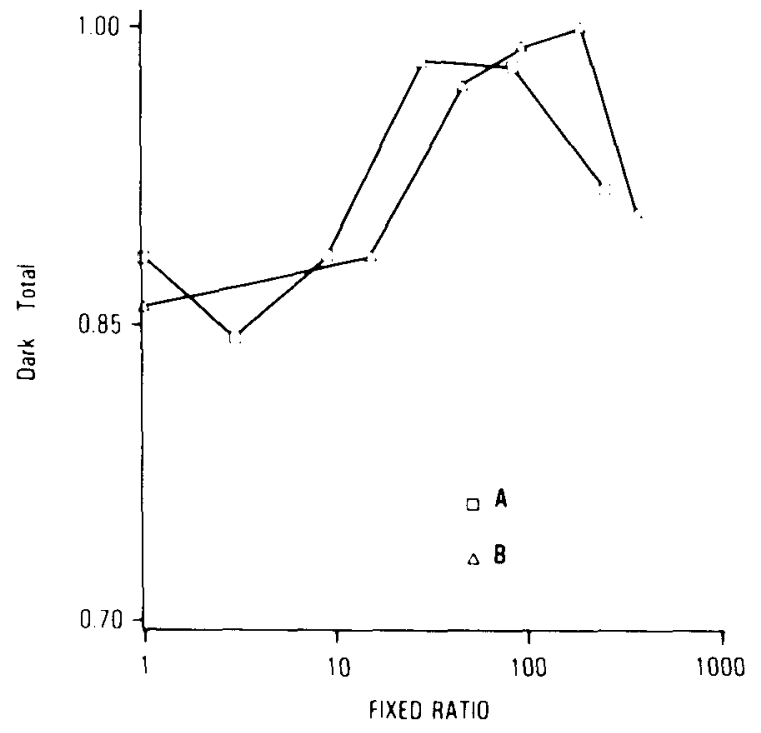

Figure 9. Mean proportion of pellets earned in the dark as a function of the fixed ratio for Groups $A$ (FR series: 1, 1, 3, 9, 27, 81, 243) and $B$ (FR series: 1, 1, 15, 45, 90, 180, 360).

cost defined as responses per reinforcer, which is often determined by an FR schedule (Hursh, 1980, 1984; Lea, 1978; Rachlin, Green, Kagel, \& Battalio, 1976). Recent work (Collier, Johnson, Hill, \& Kaufman, 1986; Hursh, Raslear, Shurtleff, Bauman, \& Simmons, 1987, in press) has shown that "price" includes more than just responses per reinforcer. A better definition of price may be a cost:benefit ratio that includes consideration of such factors as lever weight, reinforcer size, and reinforcer probability. An even more fundamental definition of price may be time expenditure per calorie of food gain. Whatever the best metric of price may be, it is clear that the FR schedule is just one contributing variable. Nevertheless, it is a powerful and consistent determiner of consumption when all other aspects of price are held constant, as they were in these experiments.

The three experiments demonstrated that the method is capable of producing highly stable and replicable demand functions within a short period of time. All three experiments produced demand functions that are qualitatively similar to functions produced under conditions that require longer periods of time. Variations in step size and order of presentation do not affect the demand functions, and demonstrate a surprising degree of invariance for behavioral data. From a practical point of view, Experiment 1 demonstrated that the use of a within-subjects design imposes problems of analysis that are easily avoided by the use of a between-subjects design within a shorter period of time: 2 weeks would be required to put two groups (6 controls and 6 experimentals) through a between-subjects study, whereas 4 weeks are required to perform an ABBA within-subjects study. In all cases, body weight was a valuable datum for interpretation of results and should be included in all future studies. Examination of the potential influence of body weight on food consumption through correlation and ANCOVA analyses, supplemented by measures of effect strength, is highly recommended. In the present experiments, such analyses indicated that, although body weight did change with manipulations of FR size and other treatments, the magnitude of such effects was insubstantial relative to the effect of FR size on food consumption. The claim that the results of closed-economy experiments are the result of uncontrolled changes in motivation (i.e., body weight or satiation) is, therefore, mistaken (Herrnstein, 1981). In Experiment 1, for instance, body-weight changes could account for approximately $6 \%$ of the changes in food consumption, while the FR size accounted for $25 \%$.

The shape of the demand curve as shown in Figures 2,6 , and 8 is curvilinear, with increasing negative slope in logarithmic coordinates (elasticity in economic terms). This basic shape has been found with both rats (Hursh et al., 1987, 1988) and monkeys (Hursh, 1984, 1986) working for food reinforcement. Hursh (1986) and Hursh et al. $(1987,1988)$ have derived an equation for demand from the assumption that elasticity is a linear function of price. That equation is consistent (i.e., accounts for more than $95 \%$ of the variance) with the form of demand curves found here and in other experiments.

\section{Pausing and Interresponse Times}

The PRP and IRT data are intended to be used as indices of motor function (Notterman \& Mintz, 1965), although other interpretations of these measures are clearly possible. To establish their validity in measures of motor function, these measures must demonstrate sensitivity to interventions that are known to alter motor function. For instance, Raslear, Leu, and Simmons (1986) examined changes in the distribution of IRTs on an FR 1 schedule of reinforcement following various doses of the organophosphate poison diisopropyl phosphorofluoridate (DFP). Many toxicants of this type are known to produce a delayed peripheral neuropathy that can result in changes in neuromuscular function and, consequently, in the gross topography of the response. They reasoned that repetitive responses, such as leverpressing in rats, might be analogous to the finger-tap test that is used in neuropsychological test batteries to diagnose neuromuscular damage (Boll, 1981; Osmon, 1981). This hypothesis was confirmed, relative to control animals, when Raslear et al. (1986) found that the IRT distributions of animals treated with a high dose of DFP exhibited changes in both central location and dispersion.

The purpose of this group of studies was not to address the validity of the PRP and IRT as motor function measures. Rather, it was designed to investigate the stability of these measures under manipulations of interest relative to the demand curves. From that perspective, it was seen that PRPs and IRTs behave in a closed economy as one would expect from the open-economy literature: pausing increases with increases in the FR, while the IRT re- 
mains relatively invariant. Similar functional relationships were obtained in all experiments, demonstrating the stability of these measures within the procedure.

A word of caution, however, should be added concerning IRTs. Although Mazur (1983), with reference to his IRT distributions, notes that "there was no tendency for the peaks to shift toward longer IRTs as ratio size increased" (p. 297), there was an increase in "long" IRTs (IRTs greater than $1 \mathrm{sec}$ ) with FR size. Such long IRTs represented less than $10 \%$ of all IRTs in the Mazur study, and it is probably true that the median IRT, as a resistant measure of central tendency (Hartwig \& Dearing, 1979), would be relatively unaffected by long IRTs in the present study. However, we have observed reliable, but small, increases in the median IRT in other work (Hursh et al., 1987,1988 ) when using many more subjects (108 rats). It is possible that the increase in IRTs with FR value is small enough to be detectable only with a large sample size.

\section{Circadian Rhythmicity}

Previous studies have demonstrated that the relative consumption of food in dark and light is useful as an index of circadian rhythmicity (Leu \& Raslear, 1983; Raslear \& Kaufman, 1983; Raslear et al., 1986). However, those studies did not vary the price of food as in the present three experiments. As was the case with PRP and IRT measures, the issue here was not one of validity but one of stability. Since the proportion of pellets eaten in the dark was reliably affected by repetition and order of presentation, extreme caution is required in the interpretation of this measure. Unlike the other measures, the functional relationship between proportion of pellets eaten in dark and FR is quite variable among experiments, and the cause of the variability is not apparent.

\section{Use as a Behavioral Tool}

Our purpose in devising a method for rapidly generating demand functions was to provide a behavioral tool for the analysis of drug effects, toxins, and other interventions of a physiological/anatomical nature. Although many behavioral techniques that can be adapted for that purpose already exist, very few techniques allow animals to be studied continuously (i.e., $24 \mathrm{~h} /$ day) over an extended period of time under motivational conditions that more closely approximate a natural environment. In a more typical behavioral study, the animals would be food deprived and would receive only a small portion of their daily food within a very short experimental session (termed an open economy by Hursh, 1984). Moreover, in the case of the most commonly used laboratory species, the rat, the experimental sessions typically occur when the animal would normally be asleep. In the present method, the animals are not food deprived and receive all of their food within their home environment (termed a closed economy), working at the times of day that are normal for the species. As Hursh (1984) has already demonstrated, the behavioral results of these two approaches to conducting a behavioral experiment can result in drastically different results. For instance, in an open economy, over a much shorter range than that used in the experiments reported here, the result of progressive increases in the price of food is a sharp decline in food intake. This contrasts with the typical result obtained in a closed economy, as demonstrated above, in which food intake is held constant over a very large price range. $\mathrm{Ob}$ viously, other important differences (in the effects of drugs, etc.) may emerge from the use of a new tool. More importantly, however, we believe that behavioral methods that more closely approximate a natural environment may reveal fundamental insights into processes that cannot otherwise be observed.

Most experimental treatments, such as drugs, have multiple actions, and, in contrast to other behavioral techniques, the present method allows for a detailed analysis of other performance indices. This added efficiency should make the method particularly desirable for screening novel treatments and interpreting behavioral effects. Since the method requires no training of subjects and is complete within 7 days, it is an extremely cost-effective behavioral tool.

\section{REFERENCES}

Aluson, J. (1983). Behavioral economics. New York: Praeger.

Bauman, R., Raslear, T. G., Hursh, S. R., Shurtleff, D., \& SimMONS, L. (1986). The effect of two series of fixed ratios on the demand for food in a closed economy. Proceedings \& Abstracts of the Annual Meeting of the Eastern Psychological Association, 57, 45.

BoLL, T. J. (1981). The Halstead-Reitan neuropsychology battery. In S. B. Filskov \& T. J. Boll (Eds.), Handbook of clinical neuropsychology. New York: Wiley.

CoHen, J. (1982). "New-look" multiple regression/correlation analysis and the analysis of variance/covariance. In G. Keren (Ed.), Statistical and methodological issues in psychology and social sciences research. Hillsdale, NJ: Erlbaum.

Collier, G. H., Johnson, D. F., Hill, W. L., a Kaufman, L. W. (1986). The economics of the law of effect. Joumal of the Experimental Analysis of Behavior, 46, 113-136.

Dixon, W. J. (1981). BMDP statistical software. Berkeley: University of California Press.

Elsmore, T. F., Fletcher, G. V., Conrad, D. G., \&odetz, F. J. (1980). Reduction of heroin intake in baboons by an economic constraint. Pharmacology, Biochemistry \& Behavior, 13, 729-732.

FrLton, M., LyON, D. O. (1966). The post-reinforcement pause. Journal of the Experimental Analysis of Behavior, 9, 131-134.

GuILFord, J. P. (1954). Psychometric methods. New York: McGrawHill.

HarTwig, F., Dearing, B. E. (1979). Exploratory data analysis. Beverly Hills: Sage Publications.

HaYs, W. L. (1963). Statistics for psychologists. New York: Holt, Rinehart and Winston.

HeRRNSTEIN, R. J. (1981). A first law for behavioral analysis. Behavioral \& Brain Sciences, 4, 392-395.

Hursh, S. R. (1980). Economic concepts for the analysis of behavior. Journal of the Experimental Analysis of Behavior, 34, 219-238.

Hursh, S. R. (1984). Behavioral economics. Joumal of the Experimental Analysis of Behavior, 42, 435-452.

Hursh, S. R. (1986, June). Factors defining open and closed economies: Mapping the continuum. In M. L. Commons (Chair), Ninth Harvard symposium on quantitative analyses of behavior. Cambridge, MA. 
HURSh, S. R., NATELSON, B. H. (1981). Electrical brain stimulation and food reinforcement dissociated by demand elasticity. Physiology \& Behavior, 26, 509-515.

Hursh, S. R., Raslear, T. G., Shurtleff, D., Bauman, R., \& SimMONS, L. (1987). The definition of price in the determination of demand for food. Proceedings \& Abstracts of the Annual Meeting of the Eastern Psychological Association, 58, 33.

Hursh, S. R., Raslear, T. G., Shurtleff, D. , Bauman, R., \& SimMONS, L. (in press). A cost-benefit analysis of demand for food. Journal of the Experimental Analysis of Behavior.

LEA, S. E. G. (1978). The psychology and economics of demand. Psychological Bulletin, 85, $441-466$.

LEU, J. R. \& Raslear, T. G. (1983). Effects of diisopropylphosphorofluoridate on circadian activity patterns in rats. Neuroscience Abstracts, 9, 1074.

MAZUR, J. E. (1983). Steady-state performance on fixed-, mixed-, and random-ratio schedules. Joumal of the Experimental Analysis of Behavior, 39, 293-307.

Notterman, J. M., Mintz, D. E. (1965). Dynamics of response. New York: Wiley.

Osmon, D. C. (1981). The use of test batteries in clinical neuropsy chology. In S. B. Filskov \& T. J. Boll (Eds.). Handbook of clinical neuropsychology. New York: Wiley.
Rachlin, H., Green, L., Kagel, J. H., Battalio, R. C. (1976) Economic demand theory and psychological studies of choice. In G. H Bower (Ed.), The psychology of learning and motivation (Vol. 10, pp. 129-154). New York: Academic Press.

Raslear, T. G., \& Kaufman, L. W. (1983). Diisopropyl phos phorofluoridate (DFP) disrupts circadian activity patterns. Neurobehavioral Toxicology \& Teratology, 5, 407-411.

Raslear, T. G., LeU, J. R., Simmons, L. (1986). The effects of diisopropyl phosphorofluoridate (DFP) on inter-response time and circadian patterns of lever-pressing in rats. Neurobehavioral Toxicology \& Teratology, 8, 655-658.

SAmUelson, P. A. (1976). Economics (10th ed.). New York: McGraw-Hill.

SAS InstituTE Inc. (1985). SAS user's guide: Statistics, Version 5 edition. Cary, NC: Author.

Watson, D. S., Holman, M. A. (1977). Price theory and its uses (4th ed.). Boston: Houghton Mifflin.

(Manuscript received September 9, 1987; revision accepted for publication January 28, 1988.) 